\title{
Numerical simulation of chloride diffusion in cementitious materials by lattice type model
}

\author{
Tianhua $\mathrm{Li}^{1}$, Zichao Pan $^{2 *}$, Wenying Bai ${ }^{1}$ and Kejia Zhang ${ }^{1}$ \\ ${ }^{1}$ Xinjiang urban construction and test Co., Ltd., 830000 Urumqi, China \\ ${ }^{2}$ Department of Bridge Engineering, Tongji University, 200092, Shanghai, China
}

\begin{abstract}
The chloride ingress is one of the most significant problems to reinforced concrete structures in coastal areas and cold regions where the de-icing salt is commonly used. In this paper, the lattice type model which has been widely used in fracture analysis of brittle materials is applied to simulate the chloride diffusion process in cementitious materials. The theoretical background of the lattice type model in solving the mass transport problem is briefly presented. The analytical solution of the Fick's law is adopted to theoretically validate the developed lattice type model. After that, two typical case studies are included to demonstrate the application of the lattice type model in the chloride ingress issue. In the first case, the tortuosity effect of the aggregates on the chloride diffusion front at meso-scale is studied by the lattice model. In the second case, the lattice model is applied in the simulation of the chloride diffusion in cracked concrete. The results show that the lattice type model can be a useful tool to simulate the chloride ingress in the cementitious materials.
\end{abstract}

\section{Introduction}

The chloride ingress is one of the most significant problems to reinforced concrete (RC) structures in coastal areas and cold regions where the de-icing salt is commonly used. The chloride ions in outside environment can transport into the cementitious materials through the connected pore structures under different mechanisms such as diffusion, convection and migration. During the chloride ingress, chloride ions gradually accumulate around the surface of rebars. When the chloride content at the surface of rebars reaches a threshold value (termed as critical chloride content [1]), the passivation layer which protect the rebar from corrosion becomes unstable and the rebar starts to corrode with other prerequisites such as water and oxygen.

To estimate the approximate initiation time of rebar corrosion induced by the chloride ingress, the analytical models are usually adopted in engineering practice due to the convenience to use $[2,3]$. The prototype of these analytical models is based on the analytical solution of the Fick's law,

$$
C(x, t)=C_{0}+\left(C_{\mathrm{s}}-C_{0}\right)\left[1-\operatorname{erf}\left(\frac{x}{2 \sqrt{D_{\text {app }} t}}\right)\right]
$$

where $C$ is the chloride content, $C_{0}$ and $C_{\mathrm{s}}$ are initial and surface chloride contents, respectively. $D_{\text {app }}$ is the apparent chloride diffusion coefficient. The word "apparent" means that the chloride binding effect has been already considered in this coefficient.
The analytical models, although simple and efficient, are obtained based on some assumptions and thus can only be applied in typical problems. Numerical simulations which are more flexible are then conducted to deal with complex situations, e.g., diffusion in cracked concrete. So far, most of the numerical simulation research is conducted at macro-scale where the concrete is considered as a homogeneous material. But at smaller scales, e.g., meso-scale, the concrete shows obvious heterogeneous characteristics. To consider the effect of the heterogeneity on material behaviors in numerical simulations, it is agreed in publications that the concrete at meso-scale should be considered as a composite material which consists of the cement paste as the matrix, the aggregate as the inclusions and the interfacial transition zone (ITZ) as the interface between the cement paste and aggregate. The analysis at meso-scale can fully consider the effect of the aggregates on the chloride ingress $[4,5]$, and thus provide a deeper insight into the mechanism of the chloride ingress.

This paper mainly presents the application of the lattice type model which is widely used in the fracture analysis of brittle materials in the analysis of the chloride diffusion problem. A comprehensive review of the lattice type model in the field of fracture mechanics can be found in [6]. Some recent researches showed that the lattice type model can be also used in the mass transport problems $[7,8]$. Based on these prior researches, this paper starts from a detailed introduction about the models and methods used in the analysis by lattice model. Then, the proposed lattice model is theoretically validated by the analytical solution of the Fick's law in

Corresponding author: z.pan@tongij.edu.cn 
both steady and transient chloride diffusion problems. After that, two case studies, i.e., chloride diffusion at meso-scale and cracked concrete, are presented to demonstrate the feasibility of the lattice type model in chloride diffusion problems.

\section{Models and methods}

\subsection{Spatial discretization}

To use the lattice type model, the continuous domain is firstly discretized into a lattice network. The first step of the discretization is to divide the domain into several squared cells with an identical size $l_{\text {cell }}$. Each cell has a squared core with an identical size $l_{\text {core }}$ located at the center of the cell. Then, the node of the lattice network is randomly generated inside the core of each cell, as shown in Fig. 1.

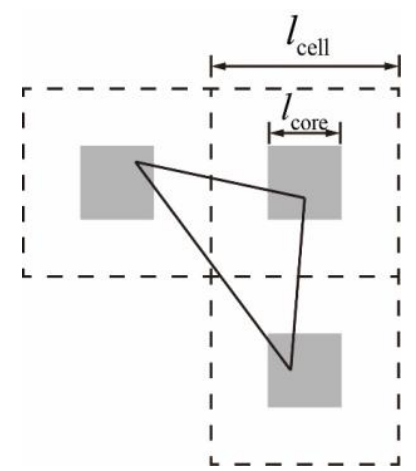

Fig. 1. Schematic of generation of node in lattice network.

After the nodes are generated, the lattice network can be formed by the method of Delaunay triangulation. Based on the size of the core inside the cell, the randomness of the lattice network can be defined as

$$
\gamma=\frac{l_{\text {core }}}{l_{\text {cell }}}
$$

Fig. 2 shows two examples of lattice networks with $\gamma=0.1$ and $\gamma=0.9$, respectively. In the fracture analysis by the lattice type model, it is assumed that the crack can only propagate along the element orientation. Thus, to eliminate the dependence of the crack pattern on the lattice network, a regular or a network with a small randomness is generally not recommended. In the chloride diffusion problem, it is assumed that chloride ions can only flow along the element orientation, which is essentially the same as that in the fracture analysis. With this consideration, the network with $\gamma=0.9$ is used throughout this paper.
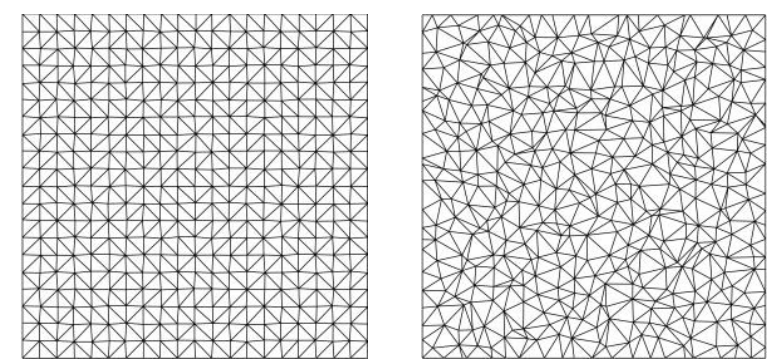
(a) $\gamma=0.1$
(b) $\gamma=0.9$

Fig. 2. Examples of lattice networks with different randomness by Delaunay triangulation.

\subsection{Governing equation}

In the lattice type model, each line segment in the network represents one element. The chloride ions are only allowed to transport along the axial direction of each element. Thus, the two-dimensional or threedimensional chloride diffusion problem is transformed into a one-dimensional (1D) problem. Based on the mass conservation law, the governing equation of the 1D chloride diffusion can be expressed as,

$$
\frac{\partial C}{\partial t}=D_{\text {app }} \frac{\partial^{2} C}{\partial x^{2}}
$$

Based on the finite element method, Eq. (3) can be finally transformed in the following matrix form for each element,

$$
\mathbf{M} \frac{\partial C}{\partial t}+\mathbf{K} C=\mathbf{f}
$$

where $\mathbf{M}$ and $\mathbf{K}$ are the element mass and diffusion matrices, respectively, $\mathbf{f}$ is a vector caused by the prescribed nodal fluxes. These matrices and vector can be calculated by

$$
\begin{gathered}
\mathbf{K}=\frac{D_{\mathrm{app}} A}{l}\left[\begin{array}{cc}
1 & -1 \\
-1 & 1
\end{array}\right] \\
\mathbf{M}=\frac{A l}{6 \omega}\left[\begin{array}{ll}
2 & 1 \\
1 & 2
\end{array}\right] \\
\mathbf{f}=\left[\begin{array}{l}
-q_{i} A \\
-q_{j} A
\end{array}\right]
\end{gathered}
$$

where $A$ and $l$ are the cross-sectional area and length of the element, respectively. $q_{i}$ and $q_{j}$ are prescribed fluxes at two nodes of an element. $A$ for each element can be determined by the Voronoi scaling method [9]. $\omega$ is a correction parameter to ensure that the total volume of the elements in the lattice network is exactly the same as that in the counterpart continuum. $\omega$ can be generally determined as

$$
\omega=\frac{\sum_{i=1}^{n} A_{i} l_{i}}{V}
$$

In most cases, however, a precise calculation of $\omega$ is not necessary. It was shown that, with enough accuracy, $\omega$ can be set as 2 and 3 for a $2 \mathrm{D}$ and $3 \mathrm{D}$ analysis, respectively [10].

\subsection{Initial and boundary conditions}

In most cases, it is reasonable to assume that the chloride content initially inside the concrete specimen is at a very low level, and thus,

$$
C(t=0)=0
$$


But if the sea sand is used, the above initial condition may not be applied. In this case, the initial chloride content should be carefully calibrated.

The boundary conditions, dependent on the surface conditions, are expressed as,

$$
\begin{gathered}
C=C_{\mathrm{s}} \text { on } \Gamma_{\mathrm{b}} \\
q=-D_{\mathrm{app}} \frac{\partial C}{\partial n} \text { on } \Gamma_{\mathrm{q}}
\end{gathered}
$$

In the analysis of chloride diffusion in a local region which is a small part of the entire concrete structure, it is often assumed that $q=0$. Thus, only the Dirichlet boundary condition, i.e., Eq. (10), is applied on the exposed surface.

\section{Theoretical validation}

The proposed lattice model to simulate the chloride diffusion problem is theoretically validated by the analytical solution of the Fick's law in both steady and transient diffusion problems. The parameters used in the validation are: (1) the size of the model is $0.5 \mathrm{~m} \times 1.0 \mathrm{~m}$; (2) the apparent chloride diffusion coefficient $D_{\text {app }}=$ $1.0 \times 10^{-11} \mathrm{~m}^{2} / \mathrm{s}$; (3) the cell size $l_{\text {cell }}=0.01 \mathrm{~m}$; (4) the randomness of the lattice network $\gamma=0.9$. The chloride ions are assumed to uniformly transport from left to the right of the model. Thus, the boundary conditions are simply $C=C_{\mathrm{s}}$ on the left side, and $q=0$ on the other three sides of the model, respectively.

The chloride diffusion process in the above case can be directly solved by the analytical solution of the Fick's law shown in Eq. (1). Thus, the lattice type model should produce the same result as the analytical solution. The comparisons between the chloride profiles in the steady and transient diffusion problems obtained by these two methods are plotted in Fig. 3 and Fig. 4, respectively. As can be seen, the chloride profile from the lattice model is the same as that from the analytical solution. Thus, the proposed lattice model is theoretically validated.

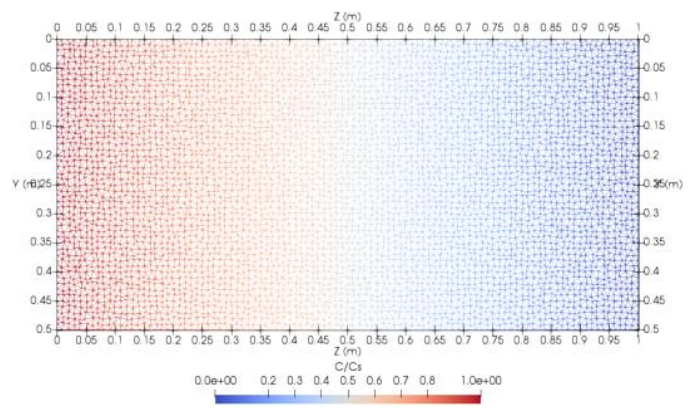

(a) chloride profile in lattice model

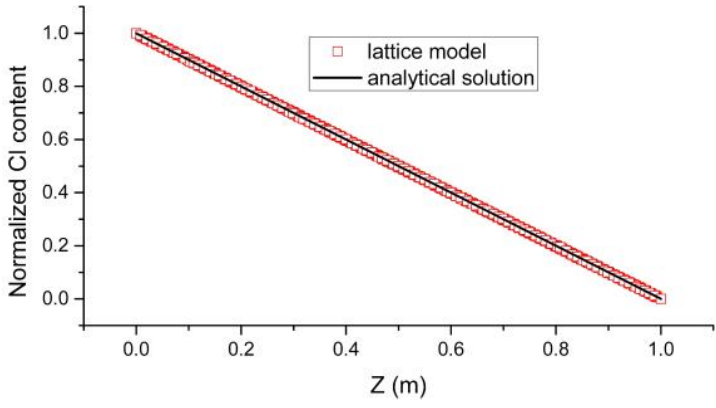

(b) comparison of chloride profile

Fig. 3. Validation of lattice model by analytical solution of Fick's law in steady chloride diffusion problem.

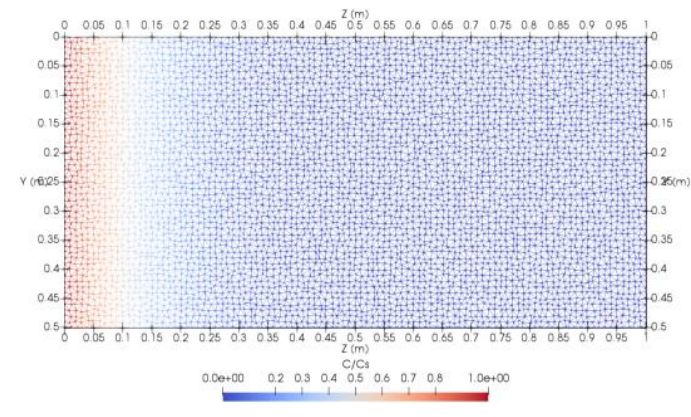

(a) contour plot, $t=50$ years

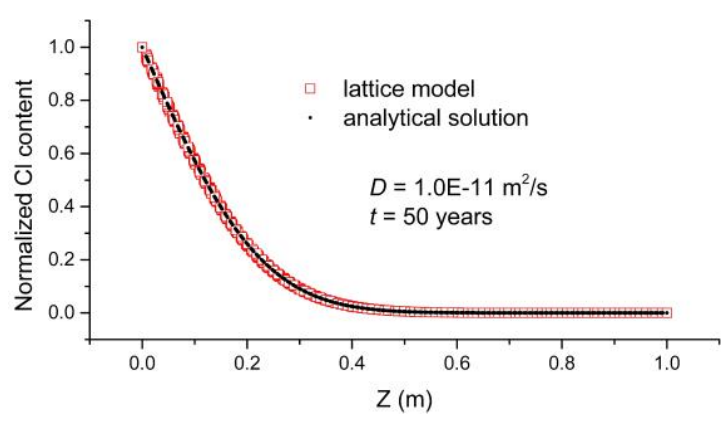

(b) chloride profile, $t=50$ years

Fig. 4. Validation of lattice model by analytical solution of Fick's law in transient chloride diffusion problem.

\section{Application}

After the lattice model is validated, in this section, two simple case studies are presented to demonstrate the application of the lattice model in the chloride diffusion problems. The first case study is to obtain the chloride diffusion front at meso-scale, which is of special importance to the design of concrete cover. The other case study is to simulate the chloride diffusion in cracked concrete to investigate the effect of cracks on the chloride diffusion front.

\subsection{Chloride diffusion front at meso-scale}

When the chloride content at the rebar surface exceeds a threshold value, the rebar will start to corrode. The threshold value is usually termed as critical chloride content, which divides the entire concrete specimen into two zones, i.e., one with a higher level of chloride content and the other with a lower level. The interface 
between these two zones is termed as the chloride diffusion front.

At macro-scale where the concrete is considered as a homogeneous material, the chloride diffusion front is simply a straight line parallel to the exposed surface. Thus, the proper thickness of the concrete cover is usually set as the depth of the chloride diffusion front at the design service life. At meso-scale, however, the chloride diffusion front has a complex shape owing to the tortuosity effect of aggregates on the chloride diffusion path. Thus, the design of concrete cover is more complicated [4].

In this case study, the aggregate shape is modelled as a circle to form the meso-scale model of concrete. Other geometries such as ellipse and polygon can be also used. Then, the meso-scale model is projected to the lattice network. The type and properties of elements in the lattice network are determined according to their positions inside the meso-scale model. As shown in Fig. 5 (a), the colours of red, yellow and blue refers to the elements of cement paste, aggregate and interface, respectively. Only the boundary condition in Eq. (10) is applied on the bottom, while the other three sides are assumed as completely isolated. During the analysis, the following parameters are used: (1) the size of the model is $0.1 \mathrm{~m} \times 0.3 \mathrm{~m}$, with the cell size $l_{\text {cell }}=1 \mathrm{~mm}$; (2) the chloride diffusivity of the cement paste $D_{\mathrm{cp}}=1.0 \times$ $10^{-12} \mathrm{~m}^{2} / \mathrm{s}$; (3) the chloride diffusivity of the interface is assumed as five times $D_{\text {cp }}[11]$; (4) the diameters of circular aggregates are $3.0 \sim 30 \mathrm{~mm}$ while the aggregates smaller than $3.0 \mathrm{~mm}$ are neglected; (5) the area fraction of aggregates is $50 \%$. The contour plot of the normalized chloride content, i.e., $C / C_{\mathrm{s}}$, at 50 and 100 years are plotted in Fig. 5(b) and (c), respectively.

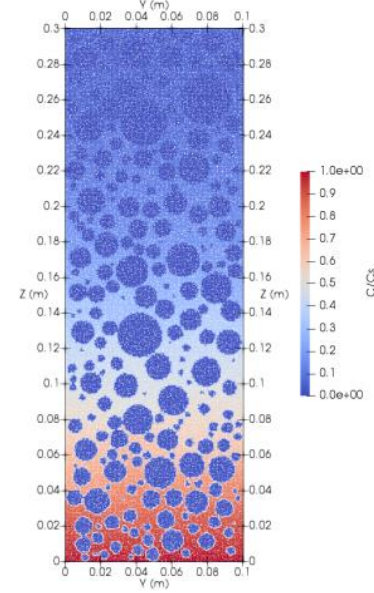

(a) chloride content at 50 years

Fig. 5. Contour plot of chloride content in mesoscopic analysis of chloride diffusion problem.

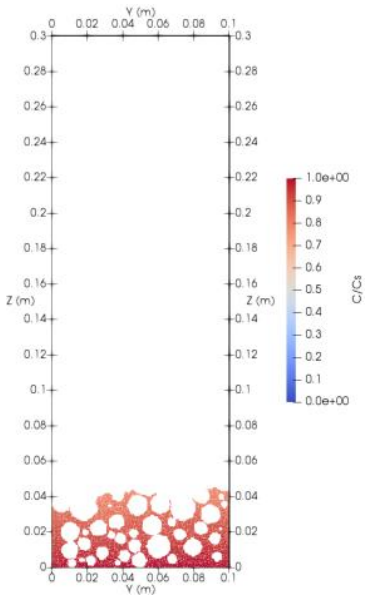

(a) $C / C_{\mathrm{r}}=0.8, t=50$ years

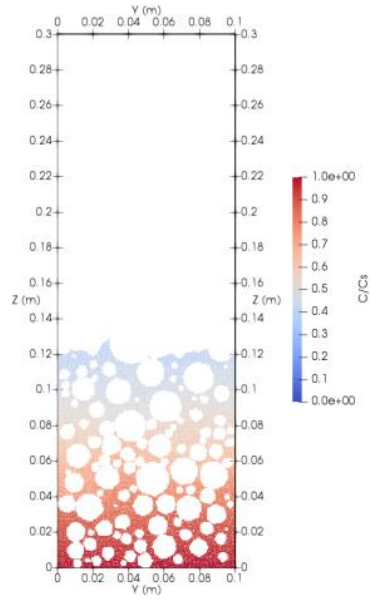

(b) $C / C_{\mathrm{r}}=0.4, t=50$ years
Fig. 6. Chloride diffusion front with different critical chloride content at meso-scale.

The critical chloride content in publications shows a high degree of variation. A literature review on this parameter can be found in $[1,12]$. In this paper, we will not discuss the absolute value of the critical chloride content. Instead, the normalized critical chloride content, i.e., $C / C_{\mathrm{r}}$, is used. Fig. 6 shows the shape of chloride diffusion front with different $C / C_{\mathrm{r}}$ at different time. Obviously, owing to the tortuosity effects of aggregates, the chloride diffusion front at meso-scale has complex shapes.

\subsection{Chloride diffusion inside cracks}

The cracks in concrete structures can accelerate the ingress of chemical substances including chloride ions, carbon dioxide, water, etc. This acceleration effect is dependent on the crack width. When the crack width is small, the chloride diffusivity inside the crack linearly increases with the crack width. When the crack width reaches a threshold value, however, the chloride diffusivity will not increase with the crack width anymore and remain a constant, as shown in Fig. 7. By an experimental study, it was found that the threshold crack width is about $0.1 \mathrm{~mm}$, while the chloride diffusivity inside the cracks when the crack width is larger than $0.1 \mathrm{~mm}$ is about $1.3 \times 10^{-9} \mathrm{~m}^{2} / \mathrm{s}$, which is $100 \sim 1000$ times the chloride diffusivity in uncracked concrete. 


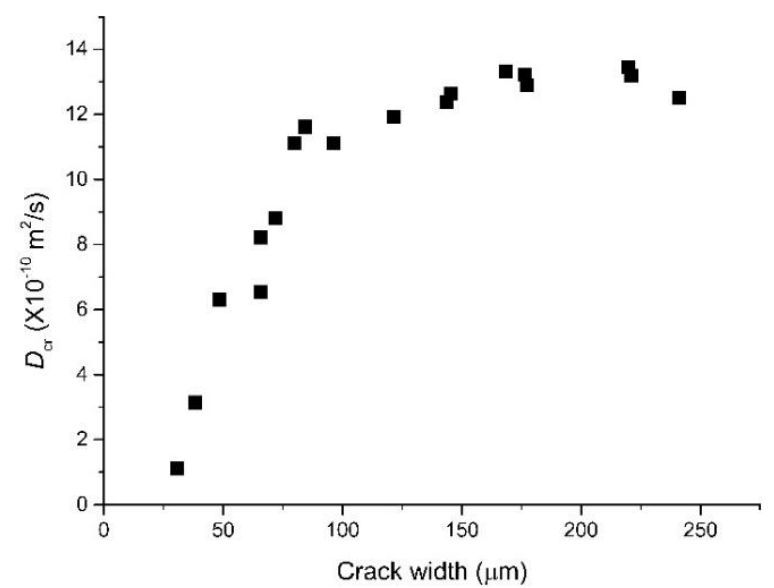

Fig. 7. Plot of chloride diffusivity in crack as a function of crack width [13].

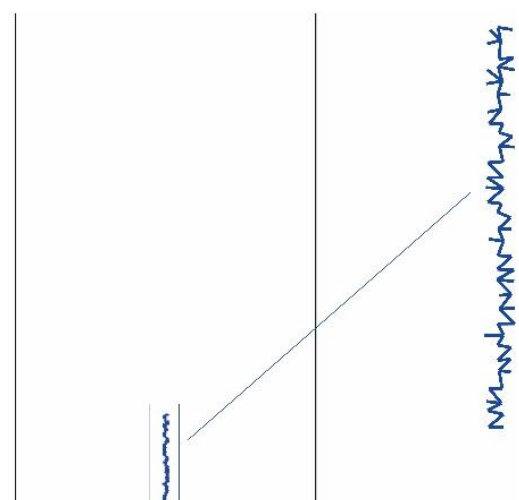

Fig. 8. Schematic of lattice elements with crack type.

To simulate the chloride diffusion in cracked concrete by lattice model, the elements with the crack type are first found according to the positions of the crack and element, as shown in Fig. 8. Then the chloride diffusivity $D_{\mathrm{r}}=1.3 \times 10^{-9} \mathrm{~m}^{2} / \mathrm{s}$ is assigned to these elements. Only as an illustration, this case study is conducted at macro-scale. For a mesoscopic analysis, the prior fracture analysis by the lattice model may be conducted to obtain the realistic, other than the artificial crack pattern. The parameters used in this case study are the same as those in the first case study on the chloride diffusion front at meso-scale.

The results of chloride content profiles and chloride diffusion front at different time are plotted in Fig. 9 and Fig. 10, respectively. As can be seen, owing to the presence of crack, the chloride diffusion front at the crack tips is deeper than that in the normal zone. By comparing Fig. 10(a) with Fig. 10(b), however, it is found that this difference gradually disappears along with the chloride diffusion process. These results indicate that the effect of cracks on the chloride diffusion may be only obvious in the early stage.

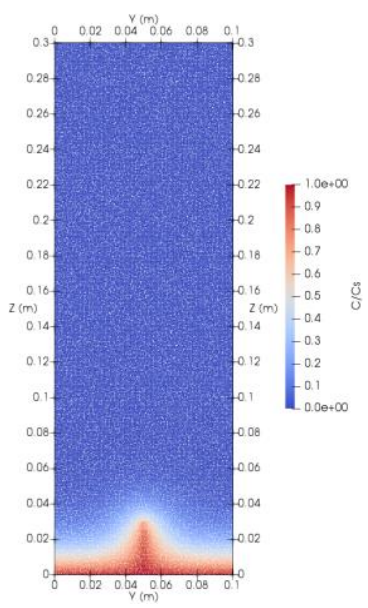

(a) 5 years

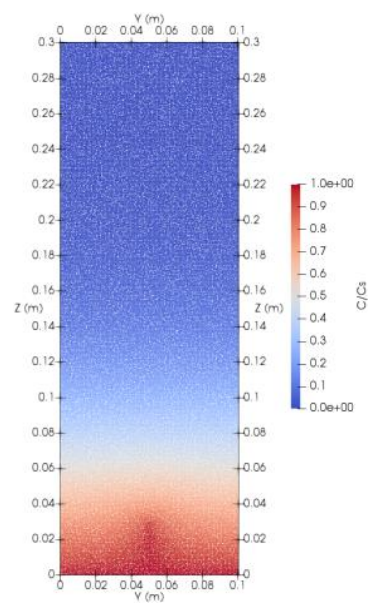

(b) 100 years
Fig. 9. Contour plot of chloride content in cracked concrete.

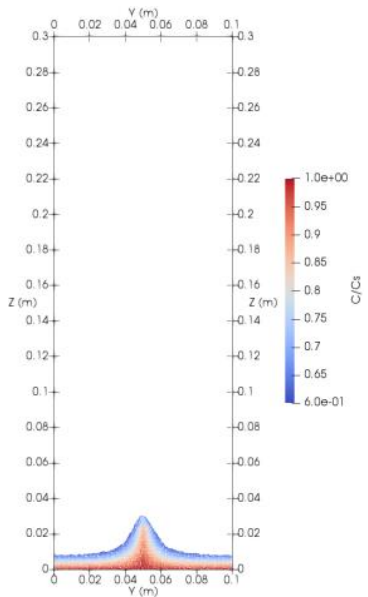

(a) 5 years

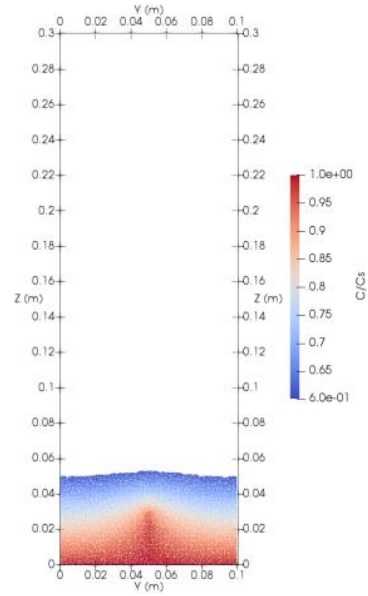

(b) 100 years
Fig. 10. Chloride diffusion front in cracked concrete.

\section{Conclusions}

In this paper, the lattice type model is applied in the analysis of the chloride diffusion problem. The general concept of the lattice model is to transform a complex global problem into a series of simple local problems. The continuous domain is discretized into a lattice network containing bar elements. Thus, 1D, 2D and 3D chloride diffusion problems can be all transformed into a $1 \mathrm{D}$ problem. The developed lattice model is theoretically validated by the analytical solution of the Fick's law. Then, two specific case studies are conducted to show the potential application of the lattice model in the chloride ingress issues. In the first case, the lattice model is used to simulate the chloride diffusion at meso-scale. The tortuosity effect of aggregates on the chloride diffusion front can be properly simulated. In the second case, the chloride diffusion in cracked concrete is simulated by the lattice model. The acceleration effect of cracks on the chloride diffusion is demonstrated. The results of the case studies show that the lattice type model can be a useful tool to simulate the chloride ingress in the cementitious materials.

The authors would like to thank the National Natural Science Foundation of China (No. 51608377) and the Fundamental 
Research Funds for the Central Universities of China for the financial support.

\section{References}

[1] U. Angst, B. Elsener, C.K. Larsen, O. Vennesland, Critical chloride content in reinforced concrete - A review, Cem. Concr. Res. 39(12) (2009) 1122-1138.

[2] S. Zhou, Analytical Model for Square Root Increase of Surface Chloride Concentration and Decrease of Chloride Diffusivity, J. Mater. Civ. Eng. 28(4) (2015).

[3] S. Zhou, Modeling chloride diffusion in concrete with linear increase of surface chloride, ACI Mater. J. 111(5) (2014) 483-490.

[4] Z. Pan, A. Chen, X. Ruan, Spatial variability of chloride and its influence on thickness of concrete cover: A two-dimensional mesoscopic numerical research, Engineering Structures 95 (2015) 154-169.

[5] Z. Pan, X. Ruan, A. Chen, Chloride diffusivity of concrete: probabilistic characteristics at meso-scale, Comput. Concr. 13(2) (2014) 187-207.

[6] Z. Pan, R. Ma, D. Wang, A. Chen, A review of lattice type model in fracture mechanics: theory, applications, and perspectives, Eng. Fract. Mech. 190 (2018) 382-409. [7] H. Sadouki, J.G.M. van Mier, Meso-level analysis of moisture flow in cement composites using a lattice-type approach, Mater. Struct. 30(10) (1997) 579-587.

[8] P. Grassl, A lattice approach to model flow in cracked concrete, Cem. Concr. Compos. 31(7) (2009) 454-460.

[9] J.E. Bolander, S. Berton, Simulation of shrinkage induced cracking in cement composite overlays, Cem. Concr. Compos. 26(7) (2004) 861-871.

[10] H. Nakamura, W. Srisoros, R. Yashiro, M. Kunieda, Time-Dependent Structural Analysis Considering Mass Transfer to Evaluate Deterioration Process of RC Structures, J. Adv. Concr. Technol. 4(1) (2006) 147-158. [11] J. Zheng, X. Zhou, Y. Wu, X. Jin, A numerical method for the chloride diffusivity in concrete with aggregate shape effect, Constr. Build. Mater. 31(6) (2012) 151-156.

[12] C. Alonso, C. Andrade, M. Castellote, P. Castro, Chloride threshold values to depassivate reinforcing bars embedded in a standardized OPC mortar, Cem. Concr. Res. 30(7) (2000) 1047-1055. 